\title{
A STUDY OF CONVERGENCE FOR A FOURTH-ORDER TWO-POINT ITERATION IN BANACH SPACES
}

\author{
J. A. Ezquerro and M. A. Hernández
}

\begin{abstract}
We present a new convergence analysis for two-point iterations by constructing a system of a prion error bounds for a fourth-order iterative method. We study the convergence of this method in Banach spaces by means of recurrence relations. This way provides some good error estimates.
\end{abstract}

\section{Introduction}

Consider the equation

$$
F(x)=0,
$$

where $F$ is a nonlinear operator from a Banach space $X$ in another Banach space $Y$. Multipoint methods are defined as iterations which use new information at a number of points. A natural generalization of the Newton method is to apply a multipoint scheme. Suppose that we already have the expressions of $F\left(x_{k}\right)$, $F^{\prime}\left(x_{k}\right)$ and $F^{\prime}\left(x_{k}\right)^{-1}$ at a current step $x_{k}$. In order to obtain a new iteration of fourth $R$-order ([8]) from Newton's method, we add one more evaluation of $F^{\prime}\left(c_{1} x_{k}+c_{2} y_{k}\right)^{-1}$, where $c_{1}$ and $c_{2}$ are real constants that are independent of $x_{k}$ and $y_{k}$, and $y_{k}$ is generated by a Newton step.

A two-point approximation scheme for all currents step $n \geq 0$ was introduced in [3] by

$$
\begin{gathered}
y_{n}=x_{n}-F^{\prime}\left(x_{n}\right)^{-1} F\left(x_{n}\right), \\
H\left(x_{n}, y_{n}\right)=F^{\prime}\left(x_{n}\right)^{-1}\left[F^{\prime}\left(x_{n}+\frac{2}{3}\left(y_{n}-x_{n}\right)\right)-F^{\prime}\left(x_{n}\right)\right],
\end{gathered}
$$

Supported in part by the University of La Rioja (grants: API-98/A25 and API-98/B12) and DGES (grant: PB96-0120-C03-02).

Keywords: Nonlinear equations in Banach spaces, fourth-order method, recurrence relations, a priori error bounds.

1991 Mathematics Subject Classification: 47H17, $65 \mathrm{~J} 15$.

Received June 22, 1998; revised May 10, 1999. 


$$
x_{n+1}=y_{n}-\frac{3}{4} H\left(x_{n}, y_{n}\right)\left[I-\frac{3}{2} H\left(x_{n}, y_{n}\right)\right]\left(y_{n}-x_{n}\right)
$$

for generating approximate solutions to (1).

We provide a new semilocal convergence theorem for two-point iteration (2) on a Banach space setting. This iteration was first introduced in [3] to approximate a locally unique solution of a nonlinear equation. Observe that (2) can be viewed as an extension of the classical Newton method. This extension is of great interest because its computational cost is low. It requires one function evaluation and one inverse at each step. This method is also cheaper to use than a two point Newton method, however the order of convergence is the same, four (see [3]).

We use some ideas considered in [4] and [5] for one-point methods that have managed to improve the convergence analysis given in [3] for the same method. These ideas consist of constructing a system of real sequences so that the convergence in Banach spaces of (2) is assured from analysing these real sequences. The use of real sequences simplifies more usual analysis of convergence for iterations in Banach spaces by means of the majorizing principle [7] or the punctual criterion [9].

In Section 2, we construct and analyse a system of real sequences, and some properties are provided. In Section 3, we establish a convergence theorem and also give an explicit expression for an error bound of method (2). Finally, a well-known integral equation is considered and error bounds are obtained.

For a more detailed information, see the relevant references [1] and [2] that deal with the monotone convergence of method (2) on a partially ordered topological space setting. In paper [1] the author has found Pták and Pták-like estimates for method (2).

\section{General recurrence relations}

Let $F$ be a nonlinear three times Fréchet-differentiable operator defined on some open convex subset $\Omega$ of a Banach space $X$ with values in another Banach space $Y$. Let $x_{0} \in \Omega$ and suppose that $\Gamma_{0}=F^{\prime}\left(x_{0}\right)^{-1} \in \mathscr{L}(Y, X)$ exists, where $\mathscr{L}(Y, X)$ is the set of bounded linear operators from $Y$ into $X$.

Assume:

(c) $\left\|\Gamma_{0}\right\|=\left\|F^{\prime}\left(x_{0}\right)^{-1}\right\| \leq \beta$,

(c) $\left\|\Gamma_{0} F\left(x_{0}\right)\right\| \leq \eta$,

(c) $\left\|F^{\prime \prime}(x)\right\| \leq M, x \in \Omega$,

(c) $\left\|F^{\prime \prime \prime}(x)\right\| \leq N, x \in \Omega$,

(c) $\left\|F^{\prime \prime \prime}(x)-F^{\prime \prime \prime}(y)\right\| \leq L\|x-y\|, \quad x, y \in \Omega, L \geq 0$. 
Let us denote $A=M \beta \eta, B=N \beta \eta^{2}$ and $C=L \beta \eta^{3}$. Define the sequences

$$
a_{0}=1=c_{0}, \quad b_{0}=\frac{2 A}{3}, \quad d_{0}=\frac{A}{2}(1+A),
$$

and

$$
\begin{gathered}
a_{n+1}=\frac{a_{n}}{1-A a_{n}\left(c_{n}+d_{n}\right)}, \quad b_{n+1}=\frac{2 A}{3} a_{n+1} c_{n+1} \\
c_{n+1}=\frac{32}{2187} \frac{27\left[4+\left(1+(3 / 2) b_{n}\right)^{2}\right] A^{3} a_{n}^{2}+18 A B a_{n}+17 C}{b_{n}^{4}\left(1+(3 / 2) b_{n}\right)^{4}} a_{n+1} d_{n}^{4} \\
d_{n+1}=\frac{3}{4} b_{n+1}\left(1+\frac{3}{2} b_{n+1}\right) c_{n+1}
\end{gathered}
$$

for all $n \geq 0$.

Based on the above, we prove the previous sequences satisfy:

$\left[\mathbf{I}_{n}\right] \quad\left\|\Gamma_{n}\right\|=\left\|F^{\prime}\left(x_{n}\right)^{-1}\right\| \leq a_{n} \beta$,

[II $\left.\mathbf{I}_{n}\right]\left\|\Gamma_{n} F\left(x_{n}\right)\right\| \leq c_{n} \eta$,

$\left[\right.$ III $\left._{n}\right]\left\|H\left(x_{n}, y_{n}\right)\right\| \leq b_{n}$,

$\left[\mathbf{I V}_{n}\right]\left\|x_{n+1}-y_{n}\right\| \leq d_{n} \eta$

$\left[\mathbf{V}_{n}\right]\left\|x_{n+1}-x_{n}\right\| \leq\left(c_{n}+d_{n}\right) \eta$,

for all $n \geq 0$. We use mathematical induction on $n$. It is easy to check the case when $n=0$ by initial hypotheses. Now we assume that $\left[\mathbf{I}_{n}\right]-\left[\mathbf{I} \mathbf{V}_{n}\right]$ are true up to a fixed $n \geq 1$.

$\left[\mathbf{I}_{n+1}\right]:$ If it is supposed that $x_{n}, x_{n+1} \in \Omega$ and $A a_{n}\left(c_{n}+d_{n}\right)<1$, then

$$
\begin{aligned}
\left\|I-\Gamma_{n} F^{\prime}\left(x_{n+1}\right)\right\| & \leq\left\|\Gamma_{n}\right\|\left\|F^{\prime}\left(x_{n}\right)-F^{\prime}\left(x_{n+1}\right)\right\| \\
& \leq a_{n} \beta M\left\|x_{n+1}-x_{n}\right\| \leq A a_{n}\left(c_{n}+d_{n}\right)<1
\end{aligned}
$$

and, by the Banach lemma, $\Gamma_{n+1}$ exists and

$$
\left\|\Gamma_{n+1}\right\| \leq \frac{\left\|\Gamma_{n}\right\|}{1-\left\|I-\Gamma_{n} F^{\prime}\left(x_{n+1}\right)\right\|} \leq \frac{a_{n} \beta}{1-A a_{n}\left(c_{n}+d_{n}\right)}=a_{n+1} \beta .
$$

$\left[\mathbf{I I}_{n+1}\right]$ : Following Argyros, Chen and Qian in [3], we have if $y_{n} \in \Omega$

$$
\begin{aligned}
F\left(x_{n+1}\right)= & \int_{0}^{1} F^{\prime \prime}\left(y_{n}+t\left(x_{n+1}-y_{n}\right)\right)(1-t) d t\left(x_{n+1}-y_{n}\right)^{2} \\
& +\int_{0}^{1}\left[F^{\prime \prime}\left(x_{n}+t\left(y_{n}-x_{n}\right)\right)(1-t)-\frac{1}{2} F^{\prime \prime}\left(x_{n}+\frac{2}{3} t\left(y_{n}-x_{n}\right)\right)\right] d t \\
& \times\left(y_{n}-x_{n}\right)^{2}
\end{aligned}
$$




$$
\begin{aligned}
& -\frac{3}{4} \int_{0}^{1}\left[F^{\prime \prime}\left(x_{n}+t\left(y_{n}-x_{n}\right)\right)-F^{\prime \prime}\left(x_{n}+\frac{2}{3} t\left(y_{n}-x_{n}\right)\right)\right] d t \\
& \times\left(y_{n}-x_{n}\right) H\left(x_{n}, y_{n}\right)\left(y_{n}-x_{n}\right) \\
& +\frac{9}{8} \int_{0}^{1} F^{\prime \prime}\left(x_{n}+t\left(y_{n}-x_{n}\right)\right) d t\left(y_{n}-x_{n}\right) H\left(x_{n}, y_{n}\right) H\left(x_{n}, y_{n}\right)\left(y_{n}-x_{n}\right)
\end{aligned}
$$

and

$$
\begin{aligned}
& \left\|\int_{0}^{1}\left[F^{\prime \prime}\left(x_{n}+t\left(y_{n}-x_{n}\right)\right)(1-t)-\frac{1}{2} F^{\prime \prime}\left(x_{n}+\frac{2}{3} t\left(y_{n}-x_{n}\right)\right)\right] d t\right\| \\
& \leq\left\|\int_{0}^{1} \int_{0}^{1}\left[F^{\prime \prime \prime}\left(x_{n}+s t\left(y_{n}-x_{n}\right)\right)-F^{\prime \prime \prime}\left(x_{n}\right)\right] d s t(1-t) d t\left(y_{n}-x_{n}\right)\right\| \\
& \quad+\frac{1}{3}\left\|\int_{0}^{1} \int_{0}^{1}\left[F^{\prime \prime \prime}\left(x_{n}+\frac{2}{3} s t\left(y_{n}-x_{n}\right)\right)-F^{\prime \prime \prime}\left(x_{n}\right)\right] d s t d t\left(y_{n}-x_{n}\right)\right\| \\
& \leq \frac{17}{216} L\left\|y_{n}-x_{n}\right\|^{2} .
\end{aligned}
$$

Hence, we get

(3) $\quad\left\|\Gamma_{n+1} F\left(x_{n+1}\right)\right\| \leq\left\|\Gamma_{n+1}\right\|\left\|F\left(x_{n+1}\right)\right\|$

$$
\begin{aligned}
\leq & \frac{1}{2} a_{n+1} \beta\left[M\left\|x_{n+1}-y_{n}\right\|^{2}\right. \\
& \left.\quad+\left(\frac{17 L}{108}+\frac{M N}{6}\left\|\Gamma_{n}\right\|+M^{3}\left\|\Gamma_{n}\right\|^{2}\right)\left\|\Gamma_{n} F\left(x_{n}\right)\right\|^{4}\right] \\
\leq & \frac{a_{n+1}}{2}\left[\frac{9 A}{16} \frac{b_{n}^{2}\left(1+(3 / 2) b_{n}\right)^{2}}{c_{n}^{2}}+\frac{17 C}{108}+\frac{A B}{6} a_{n}+A^{3} a_{n}^{2}\right] c_{n}^{4} \eta \\
= & \frac{32}{2187} \frac{27\left[4+\left(1+(3 / 2) b_{n}\right)^{2}\right] A^{3} a_{n}^{2}+18 A B a_{n}+17 C}{b_{n}^{4}\left(1+(3 / 2) b_{n}\right)^{4}} a_{n+1} d_{n}^{4} \eta \\
= & c_{n+1} \eta
\end{aligned}
$$

$\left[\mathbf{I I I}_{n+1}\right]$ :

$$
\begin{aligned}
\left\|H\left(x_{n+1}, y_{n+1}\right)\right\| & \leq\left\|\Gamma_{n+1}\right\|\left\|F^{\prime}\left(x_{n+1}+\frac{2}{3}\left(y_{n+1}-x_{n+1}\right)\right)-F^{\prime}\left(x_{n+1}\right)\right\| \\
& \leq \frac{2 A}{3} a_{n+1} c_{n+1}=b_{n+1} .
\end{aligned}
$$


$\left[\mathbf{I V}_{n+1}\right]:$

$$
\begin{aligned}
\left\|x_{n+2}-y_{n+1}\right\| & \leq \frac{3}{4}\left\|H\left(x_{n+1}, y_{n+1}\right)\right\|\left(1+\frac{3}{2}\left\|H\left(x_{n+1}, y_{n+1}\right)\right\|\right)\left\|y_{n+1}-x_{n+1}\right\| \\
& \leq \frac{3}{4} b_{n+1}\left(1+\frac{3}{2} b_{n+1}\right) c_{n+1} \eta=d_{n+1} \eta
\end{aligned}
$$

$\left[\mathbf{V}_{n+1}\right]:$

$$
\left\|x_{n+2}-x_{n+1}\right\| \leq\left\|x_{n+2}-y_{n+1}\right\|+\left\|y_{n+1}-x_{n+1}\right\| \leq\left(c_{n+1}+d_{n+1}\right) \eta .
$$

That completes the induction.

It is clear, from the above, that the following assumptions:

- $x_{n}, y_{n} \in \Omega$,

- $A a_{n}\left(c_{n}+d_{n}\right)<1$,

- $\left\{c_{n}+d_{n}\right\}$ is a Cauchy sequence

are sufficient to prove the convergence of (2).

\section{Convergence analysis}

In this section, we analyse the sequences $\left\{a_{n}\right\},\left\{b_{n}\right\},\left\{c_{n}\right\}$ and $\left\{d_{n}\right\}$ so as to prove the convergence of (2). We first need the following lemma, whose proof is trivial.

LEMMA 3.1. Let us define the real functions:

$$
f(x)=\frac{27}{16} \frac{x^{4}}{(1-(3 / 2) x(1+(3 / 4) x(1+(3 / 2) x)))^{2}}
$$

and

$$
g(x, y)=f(x)\left[1+\frac{1}{4}\left(1+\frac{3}{2} x\right)^{2}+\frac{18 A B y+17 C}{108 A^{3} y^{2}}\right]
$$

where $A, B, C>0$ and denote the real pole of $f$ by $\tau=0.4337 \ldots$ Then the following holds:

(i) $f$ is increasing for $x \in[0, \tau)$ and $f(0)=0$,

(ii) $f^{\prime}$ is increasing for $x \geq 0$ and $f^{\prime}(0)=0$,

(iii) $g(x, y)<g(x, 1)$ for $y \geq 1$,

(iv) $g_{0}(x)=g(x, 1)$ is increasing for $x \in[0, \tau)$,

(v) $g_{0}^{\prime}(x)$ is increasing for $x \in[0, \tau)$ and $g_{0}^{\prime}(0)=0$. 
Using the sequences $\left\{a_{n}\right\},\left\{b_{n}\right\},\left\{c_{n}\right\}$ and $\left\{d_{n}\right\}$ we can write

$$
b_{n+1}=g\left(b_{n}, a_{n}\right), \quad n \geq 0,
$$

where $g$ is defined in (4). Next, we give some properties of the sequences $\left\{a_{n}\right\}$, $\left\{b_{n}\right\},\left\{c_{n}\right\}$ and $\left\{d_{n}\right\}$.

Theorem 3.2. Let $A \in(0,1 / 2), B \in(0,(1 /(18 A))(p(A)-17 C))$ and $C \in$ $(0, p(A) / 17)$, where

$$
p(A)=27(A-1)(2 A-1)\left(A^{2}+A+2\right)\left(A^{2}+2 A+4\right) .
$$

Then

(i) $b_{k}>b_{k+1}$ for all $k \geq 0$,

(ii) $A_{a_{k}}\left(c_{k}+d_{k}\right)<1$ for all $k \geq 0$,

(iii) $a_{k} \geq 1$ for all $k \geq 0$,

(iv) $a_{k}<a_{k+1}$ for all $k \geq 0$.

Proof. We use induction on $k$. For $k=0$, taking into account (5), (i) is equivalent to

$$
\left(\frac{27}{16}\right) \frac{1+(1 / 4)\left(1+(3 / 2) b_{0}\right)^{2}+\left(18 A B a_{0}+17 C\right) /\left(108 A^{3} a_{0}^{2}\right)}{\left(1-(3 / 2) b_{0}\left(1+(3 / 4) b_{0}\left(1+(3 / 2) b_{0}\right)\right)\right)^{2}} b_{0}^{3}<1
$$

and it follows from the fact that $B<(1 /(18 A))(p(A)-17 C)$ and $C<p(A) / 17$. As $A<1 / 2$ and $a_{0}=1$, (iii), for $k=0$, is trivial and (ii) and (iv) are easily proved for $k=0$. Now suppose that (i)-(iv) for $k=1,2, \ldots, n-1$. By (5), Lemma 3.1 (i), (i) and (iv), for $k=n-1$, we obtain (i), for $k=n$.

The item (ii) for $k=n$ is immediate from

$$
A a_{n}\left(c_{n}+d_{n}\right)=\frac{3}{2} b_{n}\left(1+\frac{3}{4} b_{n}\left(1+\frac{3}{2} b_{n}\right)\right)
$$

and $b_{n}<\tau$, where $\tau=0.4337 \ldots$ is the real pole of $f$. Finally (iii) and (iv), for $k=n$, follow by recurrence and the induction is concluded.

THEOREM 3.3. Under the assumptions of Theorem 3.2, if we denote $\gamma=b_{2} / b_{1}$, then $\gamma<1$,

$$
b_{n}<\frac{\gamma^{4^{n-1} / 3}}{\gamma^{1 / 3}} b_{1}
$$

for all $n \geq 2, \lim _{n} b_{n}=0$ and $\sum_{n=0}^{\infty} b_{n}<+\infty$.

Proof. As $b_{2}<b_{1}$, Theorem 3.2 (i), we have $\gamma=b_{2} / b_{1}<1$. 
On the other hand, as $b_{2}=\gamma b_{1}$, from (5), it follows that $b_{3}=g\left(b_{2}, a_{2}\right)<$ $\gamma^{4} b_{2}$, since $a_{2}>a_{1}$. Next, by recurrence, we infer that $b_{n}<\gamma^{4^{n-2}} b_{n-1}$, and consequently, (7) is satisfied for all $n \geq 2$. As a result, it is clear that $\lim _{n} b_{n}=0$. Finally, from Lemma 3.1 (iv) $-(\mathrm{v})$ and $\lim _{n} b_{n}=0$, it follows that there exists a real number $\alpha \in(0,1)$ and a positive integer $n_{0}$ such that $g_{0}^{\prime}\left(b_{n}\right) \leq \alpha$, for all $n \geq n_{0}$. Thus we have $b_{n_{0}+J} \leq \alpha b_{n_{0}+J-1}, j \geq 0$, as a consequence of Lemma 3.1 (iii) and Theorem 3.2 (iv). Therefore, for $j \geq 0, b_{n_{0}+J} \leq \alpha^{J} b_{n_{0}}$ and $\sum_{n=0}^{\infty} b_{n}<$ $+\infty$.

THEOREM 3.4. Under the assumptions of Theorem 3.2, there exists $K \in \boldsymbol{R}$, $K>1$, such that $a_{n} \leq K$ for all $n \geq 0$.

Proof. We can write

$$
a_{n+1}=\prod_{i=0}^{n}\left[1+\frac{(3 / 2) b_{i}\left(1+(3 / 4) b_{i}\left(1+(3 / 2) b_{i}\right)\right)}{1-(3 / 2) b_{i}\left(1+(3 / 4) b_{i}\left(1+(3 / 2) b_{i}\right)\right)}\right]
$$

from the definition of the sequence $\left\{a_{n}\right\}$. Taking into account

$$
u(x)=\frac{1+(3 / 4) x(1+(3 / 2) x)}{1-(3 / 2) x(1+(3 / 4) x(1+(3 / 2) x))} \quad(x \geq 0)
$$

as well as the sequence $\left\{b_{n}\right\}$ is decreasing, we obtain

$$
\frac{3}{2} b_{i}<\frac{3}{2} b_{i} u\left(b_{i}\right)<\frac{3\left(2+A+A^{2}\right)}{2\left(2-2 A-A^{2}-A^{3}\right)} b_{i}
$$

for all $i \geq 0$. Then, as a consequence of the fact that $\sum_{n=0}^{\infty} b_{n}<+\infty$, it follows that

$$
\prod_{n=0}^{\infty}\left(1+\frac{3}{2} b_{n} u\left(b_{n}\right)\right)<+\infty
$$

and the proof is complete.

THEOREM 3.5. Under the assumptions of Theorem 3.2, we have

$$
c_{n}+d_{n}<\frac{3}{2 A}\left(1+\frac{A}{2}(1+A)\right) \frac{\gamma^{4^{n-1} / 3}}{\gamma^{1 / 3}} b_{1}
$$

for all $n \geq 1$ and $\gamma=b_{2} / b_{1}$.

Proof. Note that

$$
c_{n}+d_{n}=\frac{3}{2 A}\left[1+\frac{3}{4} b_{n}\left(1+\frac{3}{2} b_{n}\right)\right] \frac{b_{n}}{a_{n}} .
$$

As the function $v(x)=1+(3 / 4) x(1+(3 / 2) x)$ is increasing for all $x \geq 0$, we get 
$v\left(b_{n}\right)<1+(A / 2)(1+A)$. Hence, we have

$$
c_{n}+d_{n}<\frac{3}{2 A}\left(1+\frac{A}{2}(1+A)\right) b_{n}
$$

since $a_{n}>1$ for all $n \geq 0$. By applying Theorem 3.3, the proof is complete.

Remark. Note that $\sum_{n=0}^{\infty}\left(c_{n}+d_{n}\right)<+\infty$ follows from the previous theorem and consequently $\left\{c_{n}+d_{n}\right\}$ is a Cauchy sequence.

Due to the above analysis we are ready to prove the main convergence theorem, where we denote

$$
\overline{B(x, r)}=\{y \in X ;\|y-x\| \leq r\} \quad \text { and } \quad B(x, r)=\{y \in X ;\|y-x\|<r\} .
$$

THEOREM 3.6. Let $F$ be a nonlinear operator which is three times Fréchet differentiable in an open convex domain $\Omega$ of a Banach space $X$ with values in $a$ Banach space $Y$. Assume that:

(a) $\Gamma_{0}=F\left(x_{0}\right)^{-1}$ exists at some $x_{0} \in \Omega,\left\|\Gamma_{0}\right\| \leq \beta$ and $\left\|\Gamma_{0} F\left(x_{0}\right)\right\| \leq \eta$,

(b) $\left\|F^{\prime \prime}(x)\right\| \leq M,\left\|F^{\prime \prime \prime}(x)\right\| \leq N, \quad\left\|F^{\prime \prime \prime}(x)-F^{\prime \prime \prime}(y)\right\| \leq L\|x-y\|, \quad x, y \in \Omega$, and $\overline{B\left(x_{0}, r \eta\right)} \subset \Omega$, where $r=\lim _{n} \sum_{l=0}^{n}\left(c_{i}+d_{i}\right)$,

(c) $A \in(0,1 / 2), B \in(0,(1 /(18 A))(p(A)-17 C))$ and $C \in(0, p(A) / 17)$, where $A=M \beta \eta, B=N \beta \eta^{2}, C=L \beta \eta^{3}$ and $p(A)$ is defined in (6).

Then the sequence $\left\{x_{n}\right\}$, given by (2), is well-defined, $x_{n}, y_{n} \in B\left(x_{0}, r \eta\right)$ for all $n \geq$ 0 , and converges to a solution $x^{*} \in \overline{B\left(x_{0}, r \eta\right)}$. Moreover the solution $x^{*}$ is unique in $B\left(x_{0}, 2 /(M \beta)-r \eta\right) \cap \Omega$. Furthermore, the following error estimates hold for all $n \geq 0$ :

$$
\left\|x^{*}-x_{n}\right\| \leq \sum_{l=n}^{\infty}\left(c_{l}+d_{i}\right) \eta \leq \frac{3}{2 A}\left(1+\frac{A}{2}(1+A)\right) \frac{b_{1}}{\gamma^{1 / 3}} \sum_{l=n}^{\infty} \gamma^{4^{l-1} / 3},
$$

where $\gamma=b_{2} / b_{1}$.

Proof. Under the assumptions mentioned above, it follows immediately from the previous theorems that sequence (2) is convergent. We also have

$$
\left\|x_{n}-x_{o}\right\| \leq\left\|x_{n}-x_{n-1}\right\|+\left\|x_{n-1}-x_{n-2}\right\|+\cdots+\left\|x_{1}-x_{0}\right\| \leq \sum_{l=0}^{n-1}\left(c_{l}+d_{i}\right) \eta<r \eta \text {. }
$$

Hence, we obtain $x_{n} \in B\left(x_{0}, r \eta\right), n \geq 0$, and similarly we have $y_{n} \in B\left(x_{0}, r \eta\right)$, $n \geq 0$.

If $x^{*}$ is the limit of sequence (2), taking into account (3) and the continuity of $F$, we obtain $F\left(x^{*}\right)=0$. Indeed, observe that the sequence $\left\{c_{n}+d_{n}\right\}$ is 
decreasing as a consequence of $\left\{a_{n}\right\}$ is increasing, $\left\{b_{n}\right\}$ is decreasing and $v$ is an increasing function. Moreover, $\lim _{n}\left(c_{n}+d_{n}\right)=0$. Consequently, $\lim _{n} d_{n}=0$, since $\lim _{n} b_{n}=0$. Now, we can deduce that $\lim _{n} c_{n}=0$, and the previous assertion is true. $p \geq 0$,

On the other hand, we have $\left\|x_{n+1}-x_{n}\right\| \leq\left(c_{n}+d_{n}\right) \eta$ and consequently, for

$$
\begin{gathered}
\left\|x_{p}-x_{0}\right\| \leq\left\|x_{p}-x_{p-1}\right\|+\left\|x_{p-1}-x_{p-2}\right\|+\cdots+\left\|x_{1}-x_{0}\right\| \\
\leq\left(\left(c_{p-1}+d_{p-1}\right)+\left(c_{p-2}+d_{p-2}\right)+\cdots\left(c_{0}+d_{0}\right)\right) \eta .
\end{gathered}
$$

By letting $p \rightarrow \infty$, we obtain $\left\|x^{*}-x_{0}\right\| \leq r \eta$ and the error estimates given by (8).

Finally, to show uniqueness, let us assume that $y^{*} \in B\left(x_{0}, 2 /(M \beta)-r \eta\right) \cap \Omega$ is a solution of $F(x)=0$. According to Argyros, Chen and Qian [3]

$$
0=F\left(y^{*}\right)-F\left(x^{*}\right)=\int_{0}^{1} F^{\prime}\left(x^{*}+t\left(y^{*}-x^{*}\right)\right) d t\left(y^{*}-x^{*}\right) .
$$

Then we only have to prove that the operator $\left[\int_{0}^{1} F^{\prime}\left(x^{*}+t\left(y^{*}-x^{*}\right)\right) d t\right]^{-1}$ exists and therefore $y^{*}=x^{*}$.

\section{Application}

We obtain, applying Theorem 3.6, an a priori error bound for a well-known nonlinear integral equation. We consider the integral equation quoted in [6]:

$$
F(x)(s)=x(s)-s+\frac{1}{2} \int_{0}^{1} s \cos (x(t)) d t
$$

in the space $X=C[0,1]$ of all continuous functions on the interval $[0,1]$ with the norm $\|x\|=\max _{s \in[0,1]}|x(s)|$.

Since the three derivatives of $F$ are

$$
\begin{gathered}
F^{\prime}(u) x(s)=x(s)-\frac{s}{2} \int_{0}^{1} x(t) \sin (u(t)) d t, \\
F^{\prime \prime}(u) x y(s)=-\frac{s}{2} \int_{0}^{1} x(t) y(t) \cos (u(t)) d t, \\
F^{\prime \prime \prime}(u) x y z(s)=\frac{s}{2} \int_{0}^{1} x(t) y(t) z(t) \sin (u(t)) d t,
\end{gathered}
$$

then $M=N=L=1 / 2$.

Our next goal is to calculate $\beta$ and $\eta$. Firstly, we obtain $\left[F^{\prime}(u)\right]^{-1}$. For that, we take $x(s)=\left[F^{\prime}(u)\right]^{-1} v(s)$, i.e.

$$
x(s)=v(s)+\frac{s}{2} \int_{0}^{1} x(t) \sin (u(t)) d t .
$$


Now we solve the integral appearing in the last formula. So, we do

$$
\int_{0}^{1} v(s) \sin (u(s)) d s=\int_{0}^{1} x(s) \sin (u(s))^{\prime} d s-\int_{0}^{1} \frac{s}{2} \sin (u(s))\left[\int_{0}^{1} x(t) \sin (u(t)) d t\right] d s .
$$

Then

$$
\int_{0}^{1} x(s) \sin (u(s)) d s=\frac{\int_{0}^{1} v(s) \sin (u(s)) d s}{1-\int_{0}^{1}(s / 2) \sin (u(s)), d s}
$$

Consequently,

$$
x(s)=\left[F^{\prime}(u)\right]^{-1} v(s)=v(s)+\frac{s}{2} \frac{\int_{0}^{1} v(s) \sin (u(s)) d s}{1-\int_{0}^{1}(s / 2) \sin (u(s)), d s} .
$$

If we choose as initial iteration the function $x_{0}=x_{0}(s)=s$, we have

$$
\Gamma_{0} v(s)=\left[F^{\prime}\left(x_{0}\right)\right]^{-1} v(s)=v(s)+s \frac{\int_{0}^{1} v(s) \sin s d s}{2-\sin 1+\cos 1} .
$$

Hence

$$
\left\|\Gamma_{0}\right\| \leq \frac{3-\sin 1}{2-\sin 1+\cos 1}=\beta \quad \text { and } \quad\left\|\Gamma_{0} F\left(x_{0}\right)\right\| \leq \frac{\sin 1}{2-\sin 1+\cos 1}=\eta .
$$

Thus $A=M \beta \eta=0.314678 \ldots, \quad B=N \beta \eta^{2}=0.155867 \ldots, \quad C=L \beta \eta^{3}=0.077204 \ldots$ and the hypotheses of Theorem 3.6 are satisfied. Therefore, taking into account that our optimal upper error bounds are related to consecutive points, we consider

$$
\begin{aligned}
\left\|x^{*}-x_{n}\right\| & \leq\left(c_{n}+d_{n}\right) \eta+\sum_{t=n+1}^{\infty}\left(c_{i}+d_{i}\right) \eta \\
& \leq\left(c_{n}+d_{n}\right) \eta+\frac{3}{2 A}\left(1+\frac{A}{2}(1+A)\right) \frac{b_{1}}{\gamma^{1 / 3}} \eta \sum_{t=4^{n}}^{\infty}\left(\gamma^{1 / 3}\right)^{l}
\end{aligned}
$$

to obtain the ones shown in Table 1 .

\section{Table 1}

\begin{tabular}{|l|l|}
\hline$n$ & \multicolumn{1}{|c|}{$\left\|x^{*}-x_{n}\right\|$} \\
\hline 1 & 0.02628999751577572 \\
2 & $3.9381432 \times 10^{-7}$ \\
3 & $2.1747787 \times 10^{-26}$ \\
4 & $2.0225998 \times 10^{-103}$ \\
\hline
\end{tabular}




\title{
REFERENCES
}

[1] I. K. Argyros, A unified approach for constructıng fast two-step Newton-like methods, Monatsh. Math., 119 (1995), 1-22.

[2] I. K. Argyros AND F Szidarovszky, The Theory and Applications of Iteration Methods, CRC Press Inc., Boca Raton, 1993.

[3] I. K. Argyros, D. Chen AND Q. Qian, An Inverse-Free Jarratt Type Approxımation in a Banach Space, Approx. Theory. Appl., 12 (1996), 19-30.

[4] V Candela AND A. Marquina, Recurrence relations for rational cubic methods I: The Halley method, Computing, 44 (1990), 169-184.

[5] V Candela and A. Marquina, Recurrence relations for rational cubic methods II: The Chebyshev method, Computıng, 45 (1990), 355-367

[6] B. DöRING, Einıge sätze über das Verfahren der tangıerenden Hyperbeln in Banach-Räumen, Apl. Mat., 15 (1975), 418-464.

[ 7] L. V Kantorovich and G. P Akilov, Functional Analysis, Pergamon Press, Oxford, 1982.

[8] F. A. Potra AND V. PTÁk, Nondiscrete Induction and Iteratıve Processes, Pitman, New York, 1984.

[9] S. Shub and S. Smale, Computational complexity: on the geometry of polynomials and theory of cost, part I, Ann. Sci. Ecole Norm. Sup. (4), 18 (1985), 107-142.

\author{
UNIVERSITY OF LA RIOJA \\ Department of Mathematics and Computation \\ C/Luis de Ulloa s/N, 26004 Logroño, Spain \\ E-mail: jezquer@dmc.unirıja.es \\ mahernan@dmc.unırioja.es
}

